\title{
Session 2: Molecular biology
}

\author{
Tuesday 24 April 2001, Moderator: Dr. Hennie Hoogenboom
}

[09.00-09.30]

Cancer targeting peptides and antibodies from
phage display libraries
Hennie R. Hoogenboom
Discovery Research, Dyax B.V., Maastricht, The
Netherlands, and Maastricht University, Maastricht,
The Netherlands
Tel.: +31433871220; Fax: +31 43 3871230; E-mail:
hhoogenboom@dyax.com; URL: http://www.dyax.com

We have used phage display technology to select and affinity mature peptides, human antibodies and single domain antibody molecules with exquisite cancer specificity. For these three different types of molecules, phage libraries have been designed, made and used, and different affinity maturation strategies were applied. Examples discussed will include the derivation of anti-CEA peptides, anti-MUC-1 and MHC-peptide antibodies, and various single domain variable light chain based antibodies. Such phage-library selected peptides and proteins with anti-cancer address are expected to form the basis for more specific cancer diagnosis and treatments.

[09.30-09.50]

HuCAL - Human antibody libraries for accelerated target discovery and drug development

Elisabeth Thomassen

Research \& Development, MorphoSys AG, LenaChrist-Strasse 48, D-82152 Martinsried/Planegg, Germany

Tel.: +4989 89927 305; Fax: +49 8989927 5305;

E-mail: luig@morphosys.de

MorphoSys' proprietary HuCAL technology offers two human antibody libraries - HuCAL-scFv and HuCAL-Fab - which can be applied from target discovery to drug development. Both phage display libraries have proven to be rich sources of high-quality, functional antibodies. These could be selected on a great variety of antigens, including e.g. highly conserved proteins. The HuCAL design is optimized for high expression yields which facilitates the miniaturization and automation of the antibody selection process. The modular structure allows fast conversion into different antibody formats. The HuCAL-Fab library contains more than 10 billion functional human antibody fragments. HuCAL-Fabs are well expressed in E. coli, very stable and of high affinity. The monomeric format of a Fab fragment is ideal for additional antibody optimization making this library particularly well suited for the development of therapeutic antibodies.

\section{[09.50-10.10]}

Study of anti-HIV natural immunity by selective cloning of IgM repertoire in combinatorial library phage display vectors

R. Burioni, P.E. Varaldo and M. Clementi

Ist. Microbiologia, Università di Ancona e Dip. Scienze Biomediche, Università di Trieste, Italy

Development of antiviral strategies against HIV infection and comprehension of the virus-host interplay can not afford to lack a careful study of the role played by antibodies in the history of the disease. The importance of so-called "natural antibodies" (molecules with affinity for HIV antigens present in seronegative individuals) in protecting from the disease and in slowing the progression of the infection from the asympthomatic phase to AIDS remains still to be determined. To study this aspect of the HIV infection an original strategy for the construction of phage display combinatorial libraries containing genes coding for antibody fragments exclusively of the $\operatorname{IgM}$ and $\operatorname{IgD}$ isotype was developed. Using as a source a phage display combinatorial library containing the $\operatorname{IgM}$ repertoire of a seronegative individual genes coding for IgMFabs correspondent to natural antibodies with affinity for HIV TAT protein were selected and inserted in a novel Fab labelling expression vector (pComb3/Flag) that allowed to demonstrate and map the binding of these molecules. Sequences of the variable parts of the heavy and light chain were determined and the Fabs were demonstrated to be all identical, featuring an unusual heavy chain derived from a germ line of the Vh6a subfamily with minimal mutations. Binding to the antigen was demonstrated to be specific, being inhibited 
by antigen excess. Finally, the epitope recognized by these Fab molecules was mapped. Availability of these molecules can be important for elucidating the role of natural humoral immunity in HIV infection and disease progression.

Supported by grants to R.B. and M.C from Programma di Ricerche AIDS (Istituto Superiore di Sanità, Roma, Italy)

\section{[10.10-10.30]}

Phage display as a tool to study human autoantibodies and autoantigens in systemic autoimmune disease

J. Raats ${ }^{\mathrm{a}}$, W. Degen ${ }^{\mathrm{a}}$, S. Litjens ${ }^{\mathrm{a}}$, I. Bulduk ${ }^{\mathrm{a}}$, G. Mans $^{\text {a }}$, E. Wijnen ${ }^{\mathrm{a}}$, S. Zampieri ${ }^{\mathrm{a}}$, W. Roeffen ${ }^{\mathrm{b}}$, F. van den Hoogen ${ }^{\mathrm{c}}$ and W.J. van Venrooij ${ }^{\mathrm{a}}$

${ }^{a}$ Department of Biochemistry, University of Nijmegen, P.O. Box 9101, 6500 HB Nijmegen, The Netherlands

${ }^{\mathrm{b}}$ Department of Medical Microbiology, Section Parasitology, University Hospital of Nijmegen, P.O. Box 91016500 HB, Nijmegen, The Netherlands

${ }^{\mathrm{c}}$ Rheumatology, University Hospital of Nijmegen, P.O. Box 9101, 6500 HB, Nijmegen, The Netherlands

Selection of recombinant (auto)-antibodies specific for human autoantigens in rheumatic disease (RA, SLE, $\mathrm{SSc}$ ) from human autoimmune-patient and immunized chicken derived phage display libraries.

One important property of the immune system is its ability to discriminate between self and non-self antigens. The unresponsiveness of the immune system to self-antigens is called self-tolerance, loss of this property results in immune reactions against own or autologous antigens. Such reactions often are associated with an autoimmune disorder and eventually may contribute to clinical manifestations. The humoral immune response plays a crucial role in the onset of autoimmunity, and it is a general observation that autoimmune diseases are associated with distinct profiles of autoantibodies. During the last five years there has been a growing interest in finding possible connections between apoptosis and autoimmunity. It has been hypothesized that the recognition, uptake, processing, or presentation of modified self-antigens may promote autoantibody production. At present, many autoantigens have been found that are modified (i.e. cleaved, phosphorylated or dephosphorylated) during apoptosis. Using autoimmune patient sera in immunoprecipitation and Western blotting assays with cell extracts derived from non-apoptotic and apotptic cells, we identified sera reactivities specific for (novel) modifications of autoantigens. In this paper we give an overview of our studies on human recombinant autoantibodies derived from patients suffering from rheumatic diseases. Furthermore, we describe the generation of recombinant chicken antibodies specific for human autoantigens that will also be used to study these antigens and (apoptotic) modifications thereof in more detail. We selected human recombinant antibodies from patient phage display $\mathrm{scFv}$ combinatorial antibody libraries (complexity of $10^{7}$ or higher) derived from peripheral blood or bone marrow lymphocytes of patients with rheumatoid arthritis (RA), systemic lupus erythematosus (SLE), and scleroderma (SSc). Next to the human patient libraries we also used chicken libraries made from spleens of animals immunized with 7 human (auto)antigens simultaneously. Screening of both patient and animal derived libraries with recombinant or purified autoantigens resulted in several recombinant monoclonal human and chicken (auto)-antibodies. From our SLE patient libraries, autoantibodies against U1snRNP components U1-A, U1C, U1-70k and U1-RNA were selected. Moreover, from our SLE libraries we selected anti Ro52 and anti ribosomal $\mathrm{P}$ protein antibodies, and from both our SLE and scleroderma libraries we selected anti-La and anti $f$ Á-fodrine recombinant antibodies. From our RA patient libraries antibodies specific for RA related peptides were obtained. All human scFv clones obtained were characterized by ELISA, immunoprecipitation assays, western blotting, epitope mapping and of some clones the affinities were measured and competition experiments with patient sera were performed. Sequence analysis was performed to study the germline usage. All chicken clones were sequenced and analyzed by LIA, Western blot and ELISA. We will discuss characteristics of some of the selected scFv's i.e. epitope mapping, germline gene usage, and competition experiments with patient sera. The phage autoantibodies selected from autoimmune patient libraries were also analyzed for their specificity for (apoptotically) modified forms of their target autoantigens by Western blotting and immunprecipitation assays using apoptotic cellextracts. Some anti-La, anti-70K and anti-RA peptide scFv's recognized (apoptotically-) modified forms of their target antigens.

Conclusions: The use of antibody phage display proves to be an extremely helpful technique in studying autoantibodies and autoantigens. Modifications of autoantigens (by apoptosis and/or necrosis) seem to play a major role in the ontogeny of autoimmune diseases. Currently, by using antibody phage display libraries in combination with patient sera we continue our search for possible modifications of autoantigens involved in the ontogeny of autoimmune disease. 
Keywords: autoimmune disease, (autoimmunepatient, and immunized chicken) phage display antibody libraries, human autoantigen modification, apoptosis.

\section{[11.00-11.40]}

Construction and analyses of tetravalent antibodies Kathy Miller and Len Presta

Genentech Inc., South San Francisco, USA

As monoclonal antibodies (mAbs) become more widely used as therapeutics, we are now faced with the challenge of how to improve upon their potency. Amongst the many different approaches to this issue is the observation that the efficacy of some mAbs improves after they have been dimerized or multimerized. This has been achieved in studies either by the addition of a secondary reagent such as chemical crosslinkers, or though antibody engineering strategies. The exact mechanism by which the multimerized antibodies improve their potency is not well understood; however the increased number of binding sites on the antibody could bind and cluster more targets to amplify or diversify the cellular signal. Alternatively, the multimerized antibodies would be expected to have a higher avidity binding to its cellular target, and if the Ab engages its target longer the signaling could also be prolonged. Whatever the mechanism, many of the antibody multimerization strategies are not feasible to produce as drugs. Therefore, our goal was to engineer antibodies that contain three or four Fab, which would be capable of mimicking crosslinked antibody while still being feasible to produce. Three different forms of these constructs, which we call "Octopus" antibodies, were made, all containing human IgG1-constant domains. The heavy chain of the Octopus has tandem repeats of the $\mathrm{VH} / \mathrm{CH} 1$ domains which when expressed with the appropriate light chain, results in an antibody with a total of four Fab domains instead of the usual two of the parental IgG. Fab' 2 versions were made by removing the $\mathrm{Fc}$ region of the Octopus antibody and replacing it with a leucine-zipper. The third group of Octopus antibodies contain Fab domains independent of hinge or $\mathrm{Fc}$ regions linked end-to-end in a tandem fashion. We have constructed both three- and four-tandem Fab constructs. The Octopus antibodies were engineered to recognize three cellular targets: HER2, an EGF-like receptor that is overexpressed on many breast tumors; CD20, a tetra-span receptor expressed on B cells and B cell lymphomas; and Death Receptor 5 (DR5), a member of the TGF-family of receptors that functions to induce cellular apoptosis. The different Octopus con- structs were characterized in vitro by expression and ligand binding as well as for their ability to induce either cytostasis or cell death of various tumor cell lines. The efficacy of the Octopus antibodies in cellular internalization assays was studied as a measure of their potential to be "armed" with toxin to kill tumor cells. Mouse model studies were also done to characterize the $\mathrm{pK}$ of the various Octopus antibody forms and to analyze the potency of these antibodies in vivo.

\section{[11.40-12.00]}

Engineering of multimeric scFv antibody fragments and their application in tumour imaging and targeting.

Olan Dolezal $^{\mathrm{a}, \mathrm{b}}$, B.E. Power ${ }^{\mathrm{a}}$, A.A. Kortt ${ }^{\mathrm{a}, \mathrm{b}}$ and P.J. Hudson $^{\mathrm{a}, \mathrm{b}}$

${ }^{\mathrm{a}}$ CSIRO Health Sciences and Nutrition, 343 Royal Parade, Parkville, Victoria 3052, Australia

${ }^{\mathrm{b}}$ CRC for Diagnostic Technologies, 343 Royal Parade, Parkville, Victoria 3052, Australia

Recombinant antibody fragments can be engineered to assemble into stable multimeric oligomers of high avidity that retain parent IgG specificity to target antigens and haptens. Multimeric scFvs, such as dimer (diabodies) and trimers (triabodies), may offer an improvement over the parent antibody for tumour imaging in vivo since these smaller molecules $(60-100 \mathrm{kDa})$ allow for faster blood clearance and for rapid tumour uptake. These scFvs can also be configured as bispecific reagents for recruitment of T-cells into tumours for immunotherapy. For our model $\mathrm{scFv}$ of murine anti-influenza antibody (NC10), assembled in $V_{H}$ to $V_{L}$ orientation, we found that reducing the linker length between the domains from 5 to 0 residues forced a transition in the structure from $\mathrm{scFv}$ diabodies $[\sim 60 \mathrm{kDa}]$ to triabodies [ $80 \mathrm{kDa}$ (Atwell et al., Protein Engng. 12, 1999, 597-604). Reversal of V-domain orientation resulted in similar although not identical transition in oligomer formed. In particular, the direct ligation of $V_{L}$ to $V_{H}$ led to the formation of predominantly a tetramer (tetrabody; $100 \mathrm{kDa}$ ) rather than to an expected triabody as for $V_{H}-V_{L}$ (Dolezal et al., Protein Engng. 13, 2000, 565-574). Similar patterns of oligomerisation have been observed for other scFv fragments including the humanised anti-Lewis Y (hu3S193) monoclonal antibody. The hu3S193 scFv-5 diabody and triabody (both in $V_{H}-V_{L}$ orientation) have recently been analysed and compared with the conventional $\left.\mathrm{F}(\mathrm{ab})_{2}\right)_{2}$ fragment for targeting epithelial tumours (Power et al., submitted to Cancer Immunol. Immunotherap.; Tahtis et al., submitted to Clinical Cancer Research). Anti- 
Lewis $\mathrm{Y}$ scFv-0 $V_{L}-V_{H}$ fragment has, however, been found to form an equilibrium mixture of trimer and tetramer that cannot be easily purified from one another as was observed for NC10 scFv-0 $\left(V_{L}-V_{H}\right)$. To understand the factors influencing the transition between $\mathrm{scFv}-0$ trimers and tetramers, the crystal structure for NC10 scFv-15 fragment (Malby et al., J. Mol. Biol. 279, 1998, 901-910) has been utilised in construction of molecular models for $\mathrm{NC} 10 \mathrm{scFv}-0$ trimers and tetramers. Based on these modelling studies we predicted that steric restrictions between loops L55-L61 and L15-L17 (especially residues L15 and L57) may be responsible for the preference of four instead of three $\mathrm{scFv}$ molecules assembling to form a tetramer rather than trimer in the $\mathrm{NC} 10 \mathrm{scFv}-0 V_{L}-V_{H}$. Based on these models we have constructed and analysed several NC10 scFv-0 $V_{L}-V_{H}$ mutants with an altered tetramer versus trimer equilibrium ratio. The results of these $\mathrm{NC} 10$ mutational studies and their possible implication with respect to anti-Lewis $\mathrm{Y}$ scFv-0 $V_{L}-V_{H}$ and other such similar molecules will be presented and discussed.

[12.00-12.20]

\section{DeImmunisation $^{\mathrm{TM}}$ - Evading the immune re- sponse by $\mathbf{T}$ cell epitope removal \\ F. Carr \\ Biovation Ltd, Aberdeen, $U K$}

Over the last 20 years, various technologies have been developed to generate therapeutic monoclonal antibodies that do not elicit an adverse immune response. Techniques have ranged from the 'chimerisation' of mouse monoclonals through to the isolation of mature human antibodies from B cells. The rationale behind all these approaches is to maximize the amount of 'human' sequence based on the idea that a human protein per se will not be immunogenic. However, various clinical trials and experiments in animals have clearly demonstrated that autologous antibodies and other proteins can elicit an immune response in vivo. Work performed by Bruggerman et al. (1989) demonstrated that a monoclonal antibody from a C57/BL mouse could elicit an immune response when administered to a Balb/C mouse. More recently, clinical trials with recombinant human proteins such as 1L2 have demonstrated that such autologous proteins can elicit an immune response in up to 90 monoclonal antibodies is usually a $\mathrm{T}$ cell dependent process driven by the $\mathrm{T}$ cell epitopes present within the antibody molecule. Whilst many $\mathrm{T}$ cell epitopes from 'self' proteins induce $\mathrm{T}$ cell tolerance during ontogeny, there is evidence that the facility of other $\mathrm{T}$ cell epitopes to induce tolerance is weak (e.g. Isaacs and Walderman, Therapeutic Immunology 1, 1994, 303-312). Thus tolerance does not exist for all 'self' $\mathrm{T}$ cell epitopes. Whilst new antibodies are generated daily in the human body, these are usually produced in small quantities and in normal physiological situations such that the lack of tolerance is not a potential problem However with 'self' proteins administered in larger quantities and sometimes in abnormal physiological situations, 'self' T cell epitopes can provoke T cell responses leading to induction of antibodies. Biovation has developed DeImmunisation( to identify and eliminate $\mathrm{T}$ cell epitopes from within a protein sequence. The technique uses computer modeling of the interaction of peptides with human MHC class II molecules. Overlapping 13 mers from an antibody variable region are analyzed and scored for their binding to MHC class II. In silico predictions are performed against a wide range of MHC models representing several genotypes for each allotype and estimated to represent MHC's in approximately $96 \%$ of the world population. Following identification of MHC binding peptides, the computer approach then provides identification of single amino acids that can be changed to eliminate such binding. In this way both framework and CDR-related epitopes can he identified and eliminated. This approach to the elimination/reduction of immunogenicity has been extensively validated by means of MHC binding assays, T cell assays and mouse experiments. Data from these experiments will be presented, including results from a clinical trial with a DeImmunised antibody.

[14.00-14.40]

\section{Engineering antibodies for human therapy \\ D. Athwal Celltech Chiroscience, Slough, UK}

Since the early development of hybridoma technology, antibodies have become widely available and have been the subject of extensive research. The intrinsic properties of antibodies have resulted in them becoming widely exploited as tools in both basic research and the treatment of human disease. This, especially the latter, has been made possible through our increased understanding of antibody structure. The sequence of thousands of antibodies has been determined, and as an early example of the use of bioinformatics, the mechanism by which antibodies are able to recognize antigens was proposed. Elucidation of the three-dimensional structure of antibodies and more recently complexes of the antibody with its antigen, has enabled the structure/function relationship of antibodies to be deter- 
mined. This presentation will introduce the domain organization of antibodies and how the understanding of antibody structure, has been exploited in the design therapeutic antibodies. Data will be discussed which supports the general notion that therapies based on the use of antibodies to treat human disease have come of age. Indeed several antibodies have been approved by the FDA for the treatment of human disease. Studies carried out by the pharmaceutical industry show that total global sales of antibodies or antibody based therapeutic drug have been estimated as $\$ 1.5$ billions in the US alone. The use of naked antibodies and antibody drug conjugates will be discussed with some information on the use, progress and limitation on the use of antibody fragments in the clinic.

\section{[14.40-15.40]}

Cytokine-neutralizing antibodies selected and evolved by ribosome display from a naïve library

Lutz Jermutus, Maria Groves, Emma de Vries, Carl Matthews, Jane Osbourn

Cambridge Antibody Technology, The Science Park, Melbourn, Cambridgeshire SG8 6JJ, UK

We used biotinylated cytokine to select specifically binding $\mathrm{scFv}$ antibody fragments from a na?ve library by ribosome display. Ribosome display is an alternative display technology where in an in vitro translation step the ribosome couples mRNA to its encoded protein. Selection conditions were tailored to minimize crossreactivity and unspecific co-selection. Furthermore, we tested both error-prone and proof-reading polymerases in the amplification step of each selection cycle. Pools after three to five rounds of selection were analyzed by radio immuno assays demonstrating the enrichment of cytokine-specific binders. The pools were cloned and single clones were analyzed in a cell-based neutralization assay. The sequences of these positives will be discussed and compared to neutralizing scFvs isolated by phage display from the same naïve library. The most potent neutralizing scFvs were overexpressed in E. coli, purified to homogeneity and subsequently used for specificity and $\mathrm{IC}_{50}$ studies.

\section{[15.20-15.40]}

Identification of proteins using antibody phage display and epitope characterization

Edzard Spillner, Susanne Deckers, Thomas Grunwald and Reinhard Bredehorst

Department of Biochemistry and Molecular Biology, University of Hamburg, Germany
$\mathrm{ScFv}$-fragments represent valuable tools for detection of proteins. Here we report a novel approach of $\mathrm{scFv}$-fragments for protein identification utilizing the inherent information of the paratope for the determination of the primary structure. The high potential of phage display methods was employed to select peptides from a heptapeptide library mimicking an epitope of the protein of interest. Proof of principle is demonstrated by identification of the neuroblastom-specific surface protein NB-p260. The elucidation of the identity of NB-p260 has so far been hindered by the limited number of molecules per cell, its high molecular weight in the range of $260-280 \mathrm{kDa}$, and its instability due to proteolytic activity in the purified protein preparation. Employing our novel approach we subjected a human synthetic scFv phage display library to three rounds of selection using SDS-denatured purified NBp260. Resulting clones were tested for reactivity. Soluble scFv-fragments from positive clones was expressed and purified from periplasmic preparations by metal ion affinity chromatography. After verification of reactivity, scFv-fragments were immobilized and used for selection of a heptapeptide phage display library. From fourteen analyzed clones, three peptide sequences were obtained, two of which could not be related by conservative amino acid replacement to known proteins. The third identificated amino acid sequence resulted from several clones and could be related to $\beta$-Filamin (ABP278) after two conservative amino acid replacements. The binding of the original epitope was verified and compared to the consensus sequence on the basis of synthetic peptides.

\section{[16.10-16.50]}

Mapping of the binding site on human IgG1 for Fc receptors and design of IgG variants with improved binding

Leonard G. Presta

Genentech Inc., South San Francisco, USA

Immunoglobulin $\mathrm{G}$ (IgG) Fc receptors play a critical role in linking $\mathrm{IgG}$ antibody-mediated immune responses with cellular effector functions. The latter include release of inflammatory mediators, endocytosis of immune complexes, phagocytosis of microorganisms, antibody-dependent cellular cytotoxicity (ADCC), and regulation of immune system cell activation. A high resolution map of human IgG1 for human Fc $\gamma$ R receptors Fc $\gamma$ RI, Fc $\gamma$ RIIA, Fc $\gamma$ RIIB, and Fc $\gamma$ RIIIA as well as human FcRn has been performed. A common set of IgG1 residues is involved in binding to all Fc $\gamma \mathrm{R}$ while Fc $\gamma$ RII and Fc $\gamma$ RIII utilize distinct 
sites outside this common set. In addition to residues which abrogated binding to the $\mathrm{Fc} \gamma \mathrm{R}$, several positions were found which improved binding only to specific Fc $\gamma \mathrm{R}$ or simultaneously improved binding to one type of $\mathrm{Fc} \gamma \mathrm{R}$ and reduced binding to another type. Selected IgG1 variants with improved binding to $\mathrm{Fc} \gamma$ RIIIA were then tested in an in vitro ADCC assay and showed an enhancement in ADCC when either PBMCs or NKs were used. These variants may have important implications for using Fc-engineered antibodies for improved therapeutic efficacy.

\section{[16.50-17.20]}

Protective and crossreacting monoclonal antibodies to bacterial endotoxin

E.Th. Rietschel, D. Heumann, H. Brade, R. Barclay and F. Di Padova

Research Center Borstel, Center for Medicine and Biosciences, D-23845 Borstel, Germany

Severe sepsis end septic shock are the most serious complications of bacterial infections. Both Grampositive and Gram-negative bacteria can trigger these extreme inflammatory responses and. by so doing, cause substantial morbidity and mortality. In the United States approximately 500,000 patients suffer from septicaemia each year, and over 100,000 of these patients die despite optimal intensive care and modern antimicrobial therapy. Gram-negative bacteria are involved in about $50 \%$ of septic episodes and in their pathogenic potential, endotoxins play au important role. Endotoxins constitute macromolecular amphiphiles and are integral components of the outer membrane of Gramnegative bacteria. In this exposed position, endotoxins function as $\mathrm{O}$-antigens. They may, however. be released into the circulation expressing bioactivity and contributing to the toxic manifestations of septic shock. Chemically, endotoxins represent lipopolysaccharides (LPS) and are made up of a polysaccharide region consisting of the inner and outer cote, and a lipid A component. Structural characterization of the polysaccharide region of different LPS have revealed common structural features. The inner core region of LPS shows a high degree of similarity among Escherichia coli, Salmonella enterica and Shigella serotypes. Among a large number of broadly cross-reactive murine anti-core
LPS monoclonal antibodies (Mab) produced in our laboratories one Mah (WN1 222-5; IgG2ak) has been selected and chimerized into a human IgGlk (SDZ 219800). In ELISA and in immunoblots on purified LPS both SDZ 219-800 and WN1 222-5 show strong reactivity with all smooth LPS from $E$. coli and S. enterica serotypes. Reactivity with all the known complete core structures from E. coli (R1-R4, K-12) and Salmonella (Ra) is evident. Reactivity with Re LPS or free lipid A is not observed. These Mab cross-react with all clinical E. coli isolates from blood, urine and feces and with ether Enterobacteriaceae. Mabs SDZ 219-800 and WN1 222-5 express biological activity as they inhibit the LAL essay and the secretion of monokines (IL-6 and TNF) by mouse and human macrophages. Moreover, SDZ 219-800 and WN1 222-5 inhibit the LPSinduced release of IL-6 and TNF in vivo. Mabs SDZ 219-800 as well as WN1 222-5 neutralize the pyrogenic activity of $E$. coli LPS and protect mice from lethality in D-GalN-sensitized mice. In addition, both Mab exhibit protective activity in a murine $E$. coli 0111 infection model. Due to their broad reactivity and neutralizing characteristics, these Mab represent potential candidates for immunotherapy of patients suffering from endotoxemia and sepsis.

References: F.E. Di Padova, H. Brade, G.R. Barclay, I.R. Poxton, E. Liehl, E. Schütze. H.P. Kocher, G. Ramsay. M.H. Schreier, D.B.L. McClelland and E.Th. Rietchel, Infect. Immun. 61 (1993), 3863-3872; E. Th. Rietschel., H. Brade, O. Holst, L. Brade, S. MüllerLoennies, U. Mamat, U. Zähringer, F. Beckmann, U. Seydel, K. Brandenburg, A.J. Ulmer, T. Mattern, H. Heine, J. Schletter, H. Loppnow, U. Schönbeck, H.-D. Flad, S. Hauschildt, F.U. Schade, F.E. Di Padova and R.P.. Schumann, Curr. Top. Micro. Immun. 216 (1996), 39-81.

\section{[17.20-17.50]}

Requirements for functional antibodies in the prevention of bronchopulmonary and invasive Pseudomonas aeruginosa infection

A. Lang

Swiss Serum \& Vaccine Institute, Berne, Switzerland

Abstract not received. 